\title{
Effects of Excitotoxic Lesions of the Septum and Vertical Limb Nucleus of the Diagonal Band of Broca on Conditional Visual Discrimination: Relationship between Performance and Choline Acetyltransferase Activity in the Cingulate Cortex
}

\author{
H. M. Marston, ${ }^{1, a}$ H. L. West, ${ }^{1}$ L. S. Wilkinson, ${ }^{1}$ B. J. Everitt, ${ }^{2}$ and T. W. Robbins ${ }^{1}$ \\ Departments of ${ }^{1}$ Experimental Psychology and ${ }^{2}$ Anatomy, University of Cambridge, CB2 3EB Cambridge, United Kingdom
}

Four experiments examined the role of the cholinergic projections from the septum and vertical limb nucleus of the diagonal band of Broca (VDB) in acquisition and performance of a conditional visual discrimination. In experiments 1-3, excitotoxic lesions were made of the septum and VDB in rats using quisqualic acid, which resulted in significant reductions in ChAT activity in the hippocampus and cingulate cortex, but with no effects on cortical monoamines. In experiment 1 , there were significant impairments in acquisition of the conditional discrimination, which did not result from motivational impairments. Experiment 2 repeated these results with lesion parameters, which produced variable effects on hippocampal and cingulate ChAT activity. Those rats with reductions in predominantly cingulate ChAT were most impaired in acquisition, but those with predominantly hippocampal reductions were relatively unimpaired. Experiment 3 showed that quisquate-induced lesions of the VDB, but not of the more caudal VDB and horizontal limb nucleus of the diagonal band, produced deficits, and a model incorporating the results of experiments 1-3 showed a highly significant correlation between errors of commission and cingulate cortical ChAT activity $(r=-0.82, p<0.001)$. Experiment 4 used the excitotoxin AMPA to lesion the VDB in rats pretrained on a modified form of the conditional discrimination task. In one subgroup of rats this excitotoxin produced profound and regionally selective reductions in ChAT activity. This subgroup was also impaired in relearning the discrimination to criterion. Again, there was a significant inverse relationship between the number of errors of commission made in relearning the discrimination and cingulate ChAT activity $(r=-0.94, p<0.001)$. These experiments suggest that excitotoxic lesions of the septum/VDB produce deficits in conditional discrimination learning and performance, and that the integrity of the projection to the cingulate cortex is more crucial than that to the hippocampus in this

\footnotetext{
Received April 21, 1993; revised Aug. 30, 1993; accepted Sept. 21, 1993.

This research was supported by a Programme Grant from the Wellcome Trust H.M.M. received a studentship from the SERC. H.L.W. was supported by a Fullbright Award. We thank Dr. Janice Muir for assistance.

Correspondence should be addressed to T. W. Robbins, Department of Experimental Psychology, Downing Street, Cambridge, CB2 3EB, UK.

a Present address: Department of Pharmacology, University of Edinburgh, 1 George Square, Edinburgh, UK.

Copyright (C) 1994 Society for Neuroscience $0270-6474 / 94 / 142009-11 \$ 05.00 / 0$
}

effect. Moreover, there is a close relationship between discrimination performance and cholinergic function in the cingulate cortex. In conjunction with other results, these data suggest that different aspects of cognition and memory are modulated by cholinergic activity in different cortical regions.

IKey words: discrimination, learning, $\mathrm{ACh}$, cingulate cortex, hippocampus, septum, diagonal band]

Considerable evidence supports the contention that cholinergic mechanisms contribute to certain forms of learning and memory in humans and in experimental animals. For example, there is a significant correlation between the markers of cortical cholinergic activity, postmortem, and the degree of intellectual deficit during life in patients dying with Alzheimer's disease (Bowen et al., 1976; Perry et al., 1985). In experimental studies, some of the evidence for a role for ACh in memory and learning has depended upon the interpretation of the effects of excitotoxic lesions targeted on the nucleus basalis magnocellularis (nbm) in the rat, which provides the main extrinsic cholinergic innervation of the neocortex. Thus, ibotenate-induced lesions of the basal forebrain have been suggested to impair both working and reference memory in rats (Dunnett, 1985; Hepler et al., 1985; Murray and Fibiger, 1985; Whishaw et al., 1985) and to affect discrimination learning in nonhuman primates (Ridley et al., 1986) by damaging the cortical cholinergic projection. The strongest evidence of this type has depended on demonstrations of reversals of learning deficits by cholinergic drug treatments (e.g., Murray and Fibiger, 1985; Ridley et al., 1986).

Recent work has questioned whether all of the learning and memory deficits attributed to nbm damage are specifically related to interruption of the cortical cholinergic projection. For example, conditional discrimination learning that is profoundly impaired by ibotenate-induced lesions of the nbm is largely unaffected by quisqualate-induced lesions, which produce more severe cortical cholinergic deficits but less widespread neuronal damage at the site of the infusion of the excitotoxin in the basal forebrain (Robbins et al., 1989a). Other reference memory tasks, such as the Morris water maze (Morris et al., 1982), also show less severe deficits following AMPA-induced lesions of the basal forebrain than ibotenate-induced lesions, even though AMPA produces even greater reductions in ChAT activity in the cortex (Page et al., 1991). Moreover, working memory tasks carried out in mazes or in operant chambers similarly show deficits that are inversely related to the effectiveness of the excitotoxin in 
destroying cholinergic neurons (Etherington et al., 1987; Wenk et al., 1989; for review, see Dunnett et al., 1991). All of this evidence seriously questions the hypothesis that the cholinergic nom plays a major role in all forms of learning and memory.

The ascending cholinergic projections are not limited, however, to those arising from the nbm. There are also important projections originating in the medial septum and diagonal band of Broca, which innervate other structures implicated in certain forms of learning and memory, including the hippocampus and certain regions of limbic cortex, and which are also compromised in Alzheimer's disease and related disorders. Relatively few studies have addressed the role of these projections (but see Hagan et al., 1988; Hodges et al., 1991; Olton et al., 1991) as compared to studies of the nbm, and hardly any have considered the problems of interpretation of the effects of fiber-sparing excitotoxins. Consequently, in this study, we sought to use the same visual conditional discrimination task that was used in the previous investigations of the functions of the $\mathrm{nbm}$ (Everitt et al., 1987; Robbins et al., 1989a) for assessing the effects of excitotoxic lesions of the medial septum and the vertical limb nucleus of the diagonal band (VDB). We made lesions of these structures with the same excitotoxins, quisqualate and AMPA, that we have shown previously to damage more effectively cholinergic projections arising from the nbm (Robbins et al., 1989a, b; Dunnett et al., 1991; Page et al., 1991).

In experiments $1-3$, the excitotoxin quisqualic acid was infused at a number of sites and parameters of treatment to make lesions of the medial septum and VDB, which contain a large number of cholinergic cell bodies. In experiment 4 , based on the results obtained from the earlier experiments, we used AMPA, as a yet more potent mcans of disrupting cholinergic projections to the hippocampus and limbic cortex. In this experiment, we also changed some of the parameters of the conditional discrimination in order to gain a better understanding of the nature of the deficit produced by these lesions. In particular, the discriminative stimuli were altered, and we also studicd how the discrimination was affected by lesions of the medial septum and diagonal band when performance had already been established by prior training, in order to determine if the effects of the lesion were restricted to the learning phase.

\section{Materials and Methods}

Subjects

The animals used were male Lister hooded rats (Olac, Bicester) weighing between 180 and $270 \mathrm{gm}$ at the beginning of each experiment. They were housed, two per cagc, in a temperature-controlled room $\left(\min 22^{\circ} \mathrm{C}\right)$ under natural daylight. Limited access to food (MRC Diet 41B Laboratory chow) at the end of each day's testing resulted in animals being maintained at $90 \%$ of their initial free-feeding weight throughout the experiment.

\section{Apparatus and preliminary training}

Behavioral training and testing were carried out in six operant chambers (Campden Instruments; model 4108). Each chamber was equipped with two retractable response levers and a $0.04 \mathrm{ml}$ dipper rcinforccment system. The chambers were individually housed in sound-attenuating cabinets. Operational control, data collection, and collation were achieved by two Acorn System 3 microcomputers running inhouse software written in ONLIBASIC

The rats were initially trained preoperatively to earn food under a continuous reinforcement schedule. In experiments $1-3$, surgery was performed after this initial operant training. In experiment 4 , the animals also received conditional discrimination training (see below) prior to surgery until performance had reached asymptote at $75 \%$ correct.

\section{Surgical}

In all cases animals were anesthetized using Avertin (2,2,2-tribromoethanol in tertiary amyl alcohol, $10 \mathrm{mI} \mathrm{kg}^{-1}$ body weight, i.p.). They were administered between 5 and $10 \mathrm{ml}$ of glucose/saline $(5 \%, 0.9 \%)$ subcutaneously (s.c.) to prevent postoperative dehydration and then placed in a stereotaxic instrument (David Kopf, Tujunga, CA). A dental drill was used to remove the square flap of bone that covered the infusion sites, great care being taken not to damage the underlying sagittal sinus. The cannula array was then lowered through the sinus to the infusion site. Once the infusions were complete, the cannulas were withdrawn and a piece of hemostatic sponge (Sterispon, Allen \& Hanburys Ltd.) was inserted into the damaged sinus. The skin was sutured and dressed with antibiotic wound powder (Vetdrug, Bury St., Edmonds, UK). The animals were then placed in well-securcd, warm recovery cages for 24 hr.

Four groups of lesions were madc, targeting the cholinergic cell groups of the medial septum and the VDB.

Experiment 1. The stereotaxic coordinates (Paxinos and Watson, 1986) used were +0.3 and $0.9 \mathrm{~mm}$ to bregma, $\mathrm{L} \pm 0.4 \mathrm{~mm}, \mathrm{~V} 6.7$, and 6.2 $\mathrm{mm}$ below the exposed dura mater (incisor bar set at $2.3 \mathrm{~mm}$ below interaural line). A volume of $0.75 \mu \mathrm{l}$ or $0.12 \mathrm{~m}$ quisqualic acid (Cambridge Research Biochemicals) in phosphate buffer, $\mathrm{pH} 7.2$, was infused over $90 \mathrm{sec}$ through each of four stainless steel cannulas (30 gauge) attached via polypropylene tubing to $10 \mu \mathrm{l}$ syringes (Hamilton, Bonaduz, Switzerland) driven by an infusion pump (Harvard Apparatus, Harvard, $\mathrm{MA}$ ). The cannulas were left in place for $30 \mathrm{sec}$ before being raised to the shallower set of coordinates. A second infusion was then made and the cannulas left in place for a further $120 \mathrm{sec}$ following the infusion before being removed. Sham-operated animals received identical infusions of the phosphate buffer alone. Additional groups of sham-operated and lesioned animals were prepared for the purpose of measuring effects of the lesion on forebrain monoamines and for histological anal ysis (see below).

Experiment 2. Four infusions, at each of two depths, were made in the same manner as above at one of two sets of coordinates: 0.2 and 0.8 or 0.4 and $1.0 \mathrm{~mm}$ anterior to bregma, $\mathrm{L} \pm 0.4 \mathrm{~mm}, \mathrm{~V}-6.7$ and $-6.2 \mathrm{~mm}$; with an infusion volume of $0.5 \mu \mathrm{l}$.

Experiment 3. Four infusions were made targeting anterior and posterior regions of the VDB and the horizontal limb of the diagonal band (HDB). The coordinates used were AP 0.2 or $0.0 \mathrm{~mm}$ anterior to bregma, $\mathrm{L} \pm 0.5 \mathrm{~mm}$, and $\mathrm{V}-7.5$ and $-8.2 \mathrm{~mm}$ from dura, again with a $0.5 \mu \mathrm{l}$ infusion volume. A double cannula array was used; otherwise, the infusion parameters were the same as in experiment 1.

Experiment 4. A bilateral infusion was made of $0.5 \mu \mathrm{l}$ of a solution

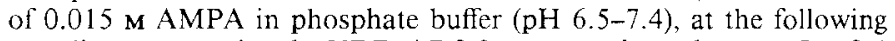
coordinates, targeting the VDB: AP $0.2 \mathrm{~mm}$ anterior to bregma, $\mathrm{L} \pm 0.4$ $\mathrm{mm}$, and V $6.7 \mathrm{~mm}$ below dura.

Postoperatively aphagia or adipsia were observed in several animals of each experimental group and these received daily injections of glucose $/$ saline ( $5 \% / 0.9 \%$ s.c.), as appropriate. A few animals died in the lesioned group in each experiment, but the remainder were able to gain weight normally on a free-feeding schedule. The animals in experiments 1 and 2 were observed to be hyperreactive to auditory and tactile stimuli. This hyperreactivity was not accompanied by vocalization and, with regular daily handling, was greatly reduced. By the third postoperative week, a naive observer had difficulty in separating lesioned from sham animals and the animals were returned to the deprivation schedule Behavioral testing generally resumed from 3-4 weeks postsurgery, consisting initially of retraining on the CRF schedule before progression to the conditional discrimination task in all experiments.

\section{Behavioral training and testing}

The conditional visual discrimination paradigm (for full description refer to Everitt et al., 1983) required the animal to make successive discriminations, of either a "fast" or "slow" flashing light provided by three $2.8 \mathrm{~W}$ bulbs situated above both levers and the magazine of the operant chamber. The animal was required to discriminate by responding on either the left or right levers following their insertion at the end of each discriminative stimulus. Correct discriminations were rewarded with $0.04 \mathrm{ml}$ of $10 \%(\mathrm{w} / \mathrm{v})$ sucrose solution; errors of omission or commission were punished with a $6 \mathrm{sec}$ period of darkness. One hundred twenty-eight trials were presented per session with an intertrial interval of $5 \mathrm{sec}$. The nature of the discriminative stimuli was identical for experiments $1-3$, but differed in experiment 4 . For the former, the 


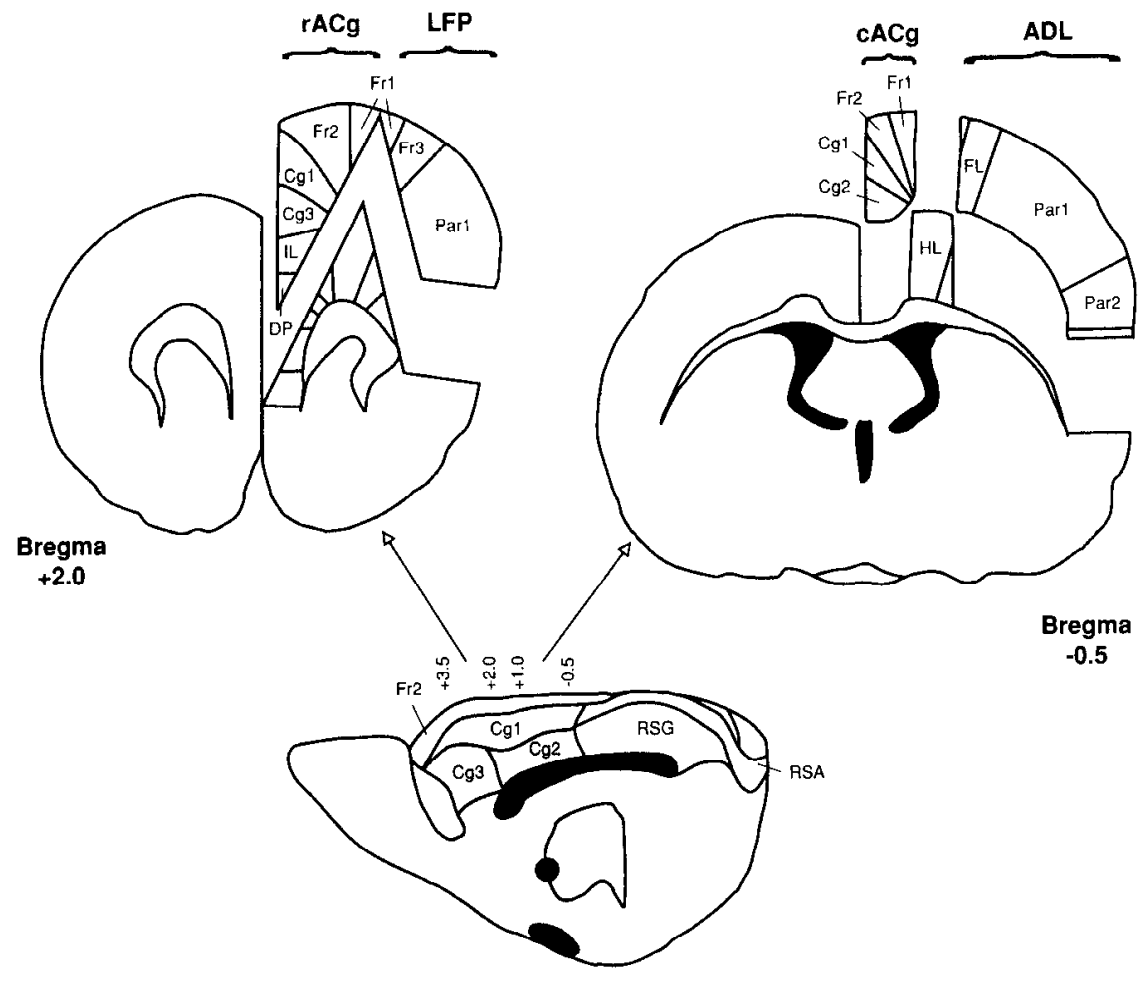

Figure 1. Schematic description of dissection and definitions of anatomical regions taken. Sections and definition of individual regions after Zilles (1985). $r A C G$, rostral anterior cingulate cortex; $C A C G$, caudal anterior cingulate cortex; $L F P$, lateral frontoparietal cortex; $A D L$, anterior dorsolateral cortex. stimuli were either 0.83 or $5 \mathrm{~Hz}$, consisting of 2 ("slow") or 12 ("fast") $0.1 \mathrm{sec}$ pulses occurring over a $2.4 \mathrm{sec}$ period, each separated by 1.1 or, alternatively, $0.1 \mathrm{sec}$, respectively (see Fig. 1 of Everitt et al., 1983). For experiment 4 , the stimuli were also 0.83 and $5 \mathrm{~Hz}$, but were doubled in duration to $4.8 \mathrm{sec}$. Furthermore, the flux of the two discriminanda was equated in the following way; the "fast" stimulus comprised 24, $0.1 \mathrm{sec}$ pulses, $0.1 \mathrm{sec}$ apart, but the "slow" stimuli consisted of four, $0.6 \mathrm{sec}$ pulses, each separated by $0.6 \mathrm{sec}$

The main indices of behavioral performance were errors of commission and errors of omission. Percentage correct was computed from those trials on which responses were made. The latency to respond following the discriminative stimulus and the latency to visit the dipper were also measured to the nearest $0.01 \mathrm{sec}$, the latter from the operation of a microswitch attached to a hinged panel giving access to the elevated reinforcement dipper. Spatial bias to one of the levers, or bias to the "slow" stimulus were measured as simple proportions of responses on the appropriate lever relative to total responses.

Acquisition was monitored in several ways: (1) errors or trials to successively stringent criteria of learning $(59 \%$, just better than chance for 128 completed trials), $67 \%, 75 \%$, and $85 \%$ correct (with less than five omissions); (2) percentage correct over sessions. In experiment 4 , the goal of the study was to examine relationships with performance factors. The main variable monitored was the number of errors committed in reattaining a performance criterion of $75 \%$ correct.

\section{Biochemical assessment of lesions}

Choline acetyltransferase (ChAT) activity. A radio-enzymatic determination of the level of ChAT activity remaining in various regions of cortex was used as the principal index for the efficacy of the septal/VDB lesions. The method used was as follows: animals were killed under ether anesthesia; the brain was rapidly removed and placed in an icecooled dissection block. Samples were dissected according to the procedures and definitions provided below. The samples were then immediately frozen at $-60^{\circ} \mathrm{C}$. All samples were later assayed in triplicate for ChAT activity after the method of Fonnum (1975).

Monoamines and their metabolites. An additional group of lesioned or sham rats, (experiment 1) was taken for measurements of monoamines and their metabolites, in order to assess the neurochemical specificity of the quisqualate-induced lesion. No behavioral training was carried out and the rats remained undeprived of food. The rats were killed 5 weeks postsurgery, and the brains dissected as described below. Levels of noradrenaline, dopamine, and 5-HT, together with the me- tabolites dihydroxyphenylacetic acid (DOPAC) and 5-hydroxyindoleacetic acid (5-HIAA), were measured using high-performance liquid chromatography, according to the methods described in detail previously by Mefford (1981) and modified by us (Jones et al., 1992).

\section{Dissection}

The definition of each sample was based on the atlas of Zilles (1985), as follows. The brain was sectioned in the coronal plane (Fig. 1). The first $2 \mathrm{~mm}$ slice, which ran from the frontal pole to a point $4.0 \mathrm{~mm}$ anterior to bregma, was discarded. The second slice was $1.5 \mathrm{~mm}$ thick. Two samples were dissected: the first, referred to as $\mathrm{rACg}$ (rostral anterior cingulate), included the pregenual cingulate cortex (Zilles $\mathrm{Cg} 1$ and $\mathrm{Cg} 3$ ) and most of the frontal region Fr2. A small section representing the boundary between frontal regions $\mathrm{Fr} 2$ and $\mathrm{Fr} 1$ was left before a second sample was taken laterally to a point approximately $2 \mathrm{~mm}$ dorsal to the rhinal fissure. This second sample, lateral frontoparietal cortex (LFP), thus effectively encompassed the remainder of the Zilles regions Fr1, Fr3, and Parl. The third slice, $1.0 \mathrm{~mm}$ in thickness, was then discarded. The last slice, running approximately from $1.5 \mathrm{~mm}$ anterior to bregma, provided the caudal anterior cingulate $(\mathrm{cACg})$ and the anterodorsolateral cortex $(A D L)$ samples. The caudal anterior cingulate cortex included postgenual cingulate cortex (comprising the Zilles regions $\mathrm{Cg} 1$ and $\mathrm{Cg} 2$ ), and $\mathrm{Fr} 2$ rostral of the hippocampus so as to exclude retrosplenial cortex. The last cortical sample, ADL, included the Zilles regions FL, HL, Par1, Par2, and a small part of GI. In experiment 4, the dissection was identical, except that an additional sample of anterior cingulate was taken from the third, previously discarded slice. This was termed intermediate, or $\mathrm{iACg}$, leaving the more posterior slice termed $\mathrm{cACg}$, as before. The hippocampus was dissected from the remaining tissue.

\section{Histological analysis of lesions}

It was not possible to assess the extent and nature of the damage produced in the lesioned group while also obtaining biochemical measures in the same animals, since the slices, described above, that were required to obtain samples of cortex impinged on the region of the septal nuclei. Consequently, a parallel group of animals with unilateral quisqualateinduced lesions was prepared for cresyl violet staining and ChAT immunocytochemistry (for details of the latter procedure, see Everitt et al., 1987). 
Table 1. Regional ChAT activity following excitotoxic lesions of the medial septum $/ \mathrm{VDB} / \mathrm{HDB}$

\begin{tabular}{|c|c|c|c|c|c|c|}
\hline & LFP & $\mathrm{rACg}$ & iACg & $\mathrm{cACg}$ & $\mathrm{ADL}$ & Hip \\
\hline \multicolumn{7}{|l|}{ Experiment 1} \\
\hline Sham & - & - & - & 2.98 & 2.48 & 4.49 \\
\hline Lesion & - & - & - & 1.91 & 2.27 & 1.53 \\
\hline$\%$ Reduction & - & - & - & -46 & -9 & -66 \\
\hline \multicolumn{7}{|l|}{ Experiment 2} \\
\hline Sham & - & - & - & 2.87 & 2.71 & 4.21 \\
\hline Cing $>$ Hipp & - & - & - & 1.18 & 2.76 & 3.62 \\
\hline$\%$ Reduction & - & - & - & -59 & 2 & -12 \\
\hline Hipp $>$ Cing & - & - & - & 2.33 & 2.84 & 1.77 \\
\hline$\%$ Reduction & - & - & - & -19 & 5 & -58 \\
\hline \multicolumn{7}{|l|}{ Experiment 3} \\
\hline Sham & 1.43 & 1.87 & - & 2.63 & 2.78 & 4.01 \\
\hline Anterior & 1.02 & 1.68 & - & 1.50 & 2.56 & 3.96 \\
\hline$\%$ Reduction & -29 & -10 & - & -43 & -8 & -2 \\
\hline Posterior & 0.86 & 1.46 & - & 1.92 & 1.53 & 2.50 \\
\hline$\%$ Reduction & -40 & -22 & - & -27 & -45 & -38 \\
\hline \multicolumn{7}{|l|}{ Experiment 4} \\
\hline Sham & 2.51 & 2.74 & 2.73 & 2.52 & - & 3.92 \\
\hline $\mathrm{ACg}$ & 1.91 & 2.54 & 1.03 & 1.02 & - & 3.01 \\
\hline$\%$ Reduction & -24 & -7 & -63 & -60 & - & -23 \\
\hline Nonselective & 1.14 & 1.92 & 0.70 & 0.71 & - & 1.83 \\
\hline$\%$ Reduction & -56 & -30 & -74 & -72 & - & -54 \\
\hline
\end{tabular}

Data are ChAT activity in the lateral frontal-parietal cortex (LFP), anterior cingulate cortex ( $\mathrm{rACg}, \mathrm{iACg}, \mathrm{cACg}$ ), anterior dorsolateral cortex (ADL), and the hippocampus (Hip). All values are expressed in micromoles of $\mathrm{ACh}$ formed/gm tissue $/ \mathrm{hr}$; SEM $<15 \%$ mean in all cases. - not determined.

\section{Data analysis}

Analysis was carried out using the GENSTAT statistical package, version 5, release 1.3 (Lawes Agricultural Trust, Rothamstead Experimental Station, Hertfordshire, UK). Inspection of residual versus fitted value plots was used to confirm that the assumptions of the analysis had been satisfied. Appropriate transformations werc used when required. Post

Table 2. Regional monoamine levels following quisqualate-induced lesions of the medial septum

\begin{tabular}{|c|c|c|c|c|}
\hline & $\mathrm{rACg}$ & $\mathrm{CACg}$ & $\mathrm{ADL}$ & Hip \\
\hline \multicolumn{5}{|c|}{ Noradrenaline } \\
\hline Sham & 0.27 & 0.39 & 0.27 & 0.32 \\
\hline Lesion & 0.31 & 0.35 & 0.25 & 0.28 \\
\hline \multicolumn{5}{|l|}{ DOPAC } \\
\hline Sham & 0.04 & 0.05 & 0.03 & 0.01 \\
\hline Lesion & 0.03 & 0.02 & 0.01 & 0.01 \\
\hline \multicolumn{5}{|c|}{ Dopamine } \\
\hline Sham & 0.09 & 0.05 & 0.03 & 0.02 \\
\hline Lesion & 0.06 & 0.03 & 0.01 & 0.01 \\
\hline \multicolumn{5}{|l|}{ 5-HIAA } \\
\hline Sham & 0.44 & 0.35 & 0.23 & 0.38 \\
\hline Lesion & 0.39 & 0.24 & 0.16 & 0.31 \\
\hline \multicolumn{5}{|c|}{ Homovanillic acid } \\
\hline Sham & 0.09 & 0.05 & 0.05 & 0.04 \\
\hline Lesion & 0.08 & 0.04 & 0.02 & 0.04 \\
\hline \multicolumn{5}{|l|}{$5-\mathrm{HT}$} \\
\hline Sham & 0.51 & 0.37 & 0.28 & 0.25 \\
\hline Lesion & 0.56 & 0.34 & 0.25 & 0.24 \\
\hline
\end{tabular}

$\mathrm{rACg}$ and $\mathrm{CACg}$, rosiral and caudal anterior cingulate cortex; $\mathrm{ADL}$, anterior dorsal lateral cortex; Hip, dorsal hippocampus. All values expressed as $\mathrm{ng} / \mathrm{mg}$ tissue (wet weight). All SEMs $<15 \%$ of the mean. hoc comparison of means derived were tested with Sidak's multiplicative $t$ tables, a conservative formulation based upon the number of comparisons, $k$, made. Regressions were based upon a linear model and calculated by the least-squares technique. Step forward regression based on the intercorrelation matrix of partial correlations of Pcarson's rho for biochemical measures of ChAT activity and selected behavioral variables was also undertaken.

\section{Results}

Experiment 1: effects of quisqualate-induced lesions of the septum/VDB

\section{IIistological analysis}

Analysis of the effects of unilateral lesions of the septum/VDB made with quisqualic acid in the parallel group prepared for histological analysis revealed a reduction in the number of ChATimmunoreactive neurons in the region of the excitotoxin infusion, as compared to the saline-infused side, both in the medial septal nucleus and in the dorsal portions of the rostral VDB. In cresyl violet-stained sections, there was clear evidence of neuronal loss in both the medial and lateral septum, as well as in dorsal parts of the rostral VDB and with some gliosis in the region of neuronal loss. A large increase in the size of the lateral ventricles was evident in many brains sustaining these septal/ VDB lesions.

\section{Biochemical results}

ChAT activity. Eight sham and 11 lesioned animals were assayed for ChAT activity in three regions of cortex: $\mathrm{cACg}$, anterodorsal cortex, and the hippocampus. As shown in Table 1, ChAT activity was reduced by about $66 \%$ in the hippocampus and $46 \%$ in the $\mathrm{CACg}$. The ANOVA revealed a group by regions interaction $[F(3,51)=44.19, p<0.001]$, post hoc analysis revealing that the interaction was the result of significant reductions in 


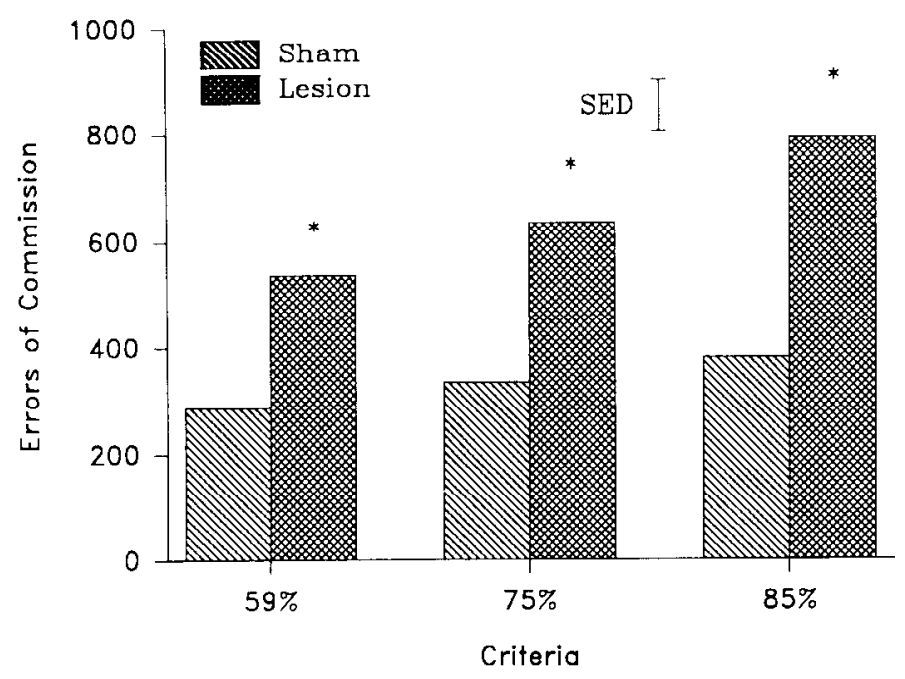

Figure 2. Experiment 1. Mean cumulative errors of commission for the septum/VDB lesion and sham control groups made in attaining each of the criteria of acquisition of the conditional visual discrimination. Fifty-nine percent is just better than chance; $85 \%$ generally represents mean asymptotic performance. The vertical bar depicts 1 standard error of the difference between the means (SED) obtained from the groups $x$ criteria interaction. This value forms the denominator of a $t$ ratio and provides a visual index for making pairwise comparisons between the groups. The asterisk indicates significant group differences at at least the $5 \%$ level.

the level of ChAT activity in hippocampus and $\mathrm{CACg}$, but with no significant reduction in the level in the ADL of the lesioned animals.

Monoamines and metabolites. There were no significant effects of the quisqualate-induced lesion on levels of any of the monoamines or metabolites measured in the cortex (Table 2), suggesting that the lesion of the septum had not resulted in damage to monoaminergic fibers of passage in the vicinity of the septum.

\section{Behavioral results}

Postoperative recovery. There were no differences in the body weights of the two groups (lesion, $258 \mathrm{gm}$; sham, $271 \mathrm{gm}$ ). When returned to the CRF schedule there were no differences in the rate $[9.8,12.3$ responses per minute (rpm)] or bias of responding ( $53.6 \%, 56.8 \%$ to the left) between the two groups.

Acquisition of conditional visual discrimination. The attainment of three performance criteria ( $59 \%, 75 \%$, and $85 \%$ correct; less than five omissions) was quantified in terms of the cumulative errors of commission. Figure 2 shows that the lesioned animals were impaired in the acquisition of the discrimination to all three of the performance criteria. Analysis revealed two main effects, of group $[F(1,17)=6.21, p=0.024]$ and of criterion $[F(2,34)=15.07, p<0.001]$. The interaction between group and criterion failed to reach significance $[F(2,34)=3.05, p=$ $0.061]$. Analysis of the total number of omissions that occurred infrequently, even when restricted to the least stringent criterion, revealed no significant difference between the two groups $[F(1,17)$ $=0.26, p=0.62 \mathrm{]}$. The lesion had no differential effect on correct latency, magazine latency, or lever bias indices. Analysis of correct latency revealed no main cffect of group $[F(1,17)=0.57$, $p=0.46]$ or interaction between group and criterion $[F(2,34)$ $=0.61, p=0.55]$. Similarly, neither the group $[F(1,17)=0.29$, $p=0.60]$ nor the interaction terms $[F(2,34)=1.07, p=0.35]$

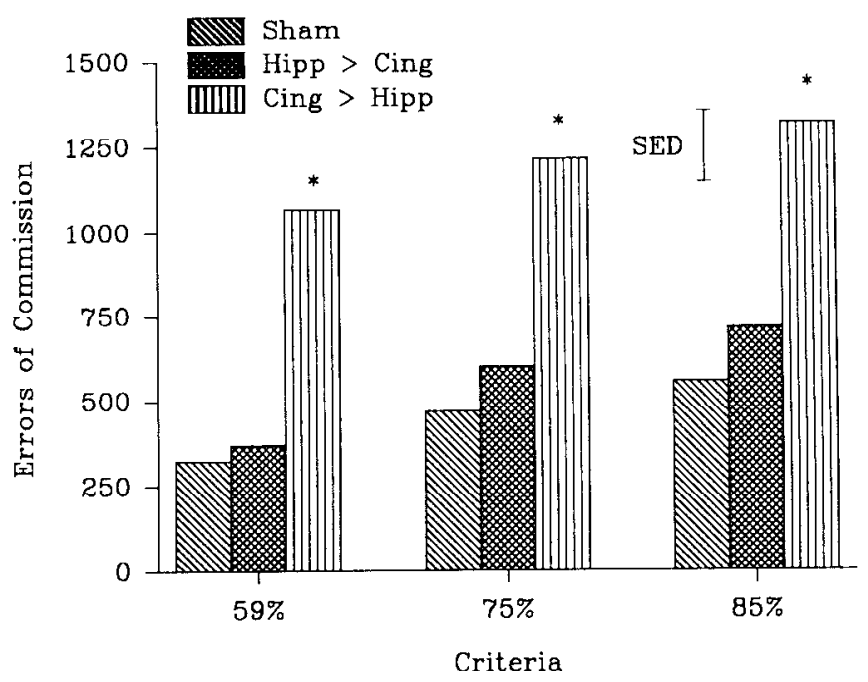

Figure 3. Experiment 2: mean cumulative errors of commission made in attaining each of the criteria of acquisition of the conditional visual discrimination for the sham control group and for two groups with ChAT reductions following septum/VDB lesions. Fifty-nine percent is just better than chance; $85 \%$ generally represents mean asymptotic performance. The vertical bar depicts 1 standard error of the difference $(S E D)$ between the means obtained from the groups $\times$ criteria interaction. The Hipp > Cing group had greater reductions in ChAT activity in the hippocampus than in the cingulate cortex: the Cing > Hipp group showed the reverse pattern. See Figure 2 caption for further explanation.

for magazine latency were significant; nor were those for lever bias [group, $F(1,17)=0.27, p=0.56$ ].

Experiment 2: effects of quisqualate-induced lesions of the septum/VDB using two sets of cannula placements

\section{Biocherrical results}

Inspection of the biochemical data revealed heterogeneity in the pattern of reductions of ChAT activity in the cingulate cortex and the hippocampus, with depletions in primarily one structure or the other. Consequently, animals were assigned to two subgroups post hoc on this basis (Table 1). The allocation of the animals to the two subgroups was supported by a significant group by region interaction $[F(4,30)=12.99, p<0.001]$. Post hoc tests confirmed that the level of hippocampal ChAT activity in the Hipp>Cing subgroup $(n=7)$ was significantly less than that of the Cing $>$ Hipp subgroup $(p<0.005)$. Conversely, ChAT activity in the $\mathrm{CACg}$ in the Cing $>$ Hipp subgroup $(n=5)$ was significantly less than that found in the Hipp $>$ Cing subgroup $(p<0.005)$. Both subgroups showed significant reductions in ChAT activity in the $\mathrm{cACg}(\mathrm{cACg})$ and in the hippocampus, compared with sham-lesioned controls $(n=6)$. However, neither group revealed a significant change in the level of ChAT activity in the ADL, compared with shams.

\section{Behavioral results}

Postoperative recovery. Ten days after surgery there were no significant differences in the weights of the two groups (lesion, $264 \mathrm{gm}$; sham, $273 \mathrm{gm})$. When returned to the CRF schedule, measures of rate (means, $10.1,13.8 \mathrm{rpm}$ ) and bias $(46.2,56.6 \%$ to the left) of responding were equivalent between the lesion and sham groups.

Acquisition of conditional visual discrimination. As seen in Figure 3, the two lesion subgroups were distinguishable in terms of the cumulative errors of commission [group effect, $F(2,15)$ 

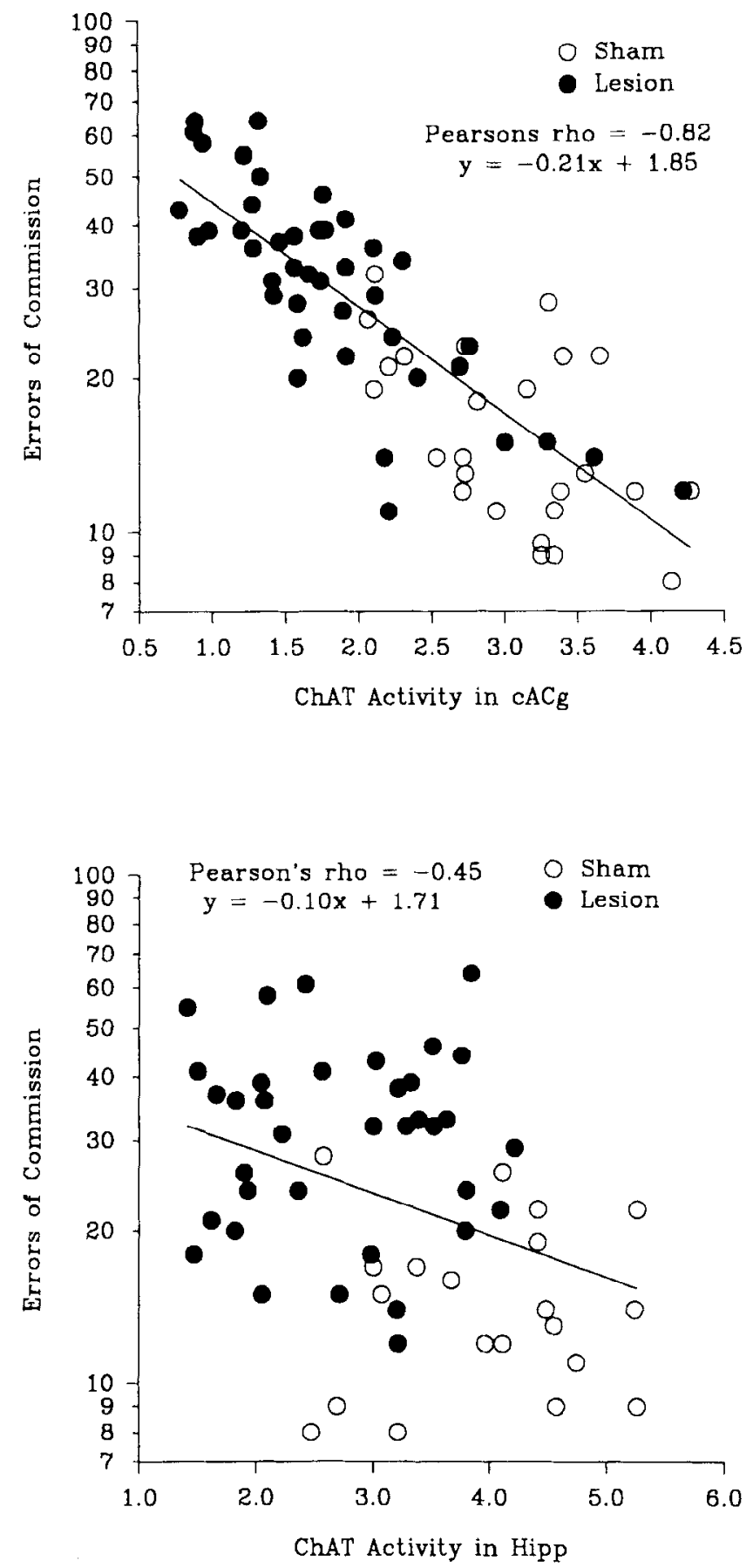

Figure 4. Log linear regression of errors of commission and ChAT activity in the $\mathrm{CACg}$ (top) and hippocampus (bottom) for experiments 1-3. Each point represents an individual rat. Also shown are the equations of the best-fitting line and values for Pearson's $r$.

$=13.62, p<0.001]$. Post hoc analysis confirmed that the Cing $>$ Hipp group made significantly more errors $[t(30)=4.59$, $p<0.01]$ than either the sham or Hipp $>$ Cing groups $[t(30)=$ $3.90, p<0.01]$. However, the Hipp $>$ Cing group was indistinguishable from the sham group in terms of errors $[t(30)=0.69$, $p>0.10]$. There were no differences between the groups in any of the other task variables, including omissions, correct latency, or spatial response bias [all $F(2,15)<1.2, p>0.05$ ]. There was a small lengthening of magazine latency in the Hipp $>$ Cing group, but this did not attain significance [group effect, $F(2,15)=2.56$, $p=0.110]$.

\section{Experiment 3: effects of quisqualate-induced lesions of the caudal VDB}

Biochemical results

Lesions to the caudal VDB, including parts of the horizontal diagonal band in many cases, produced a significant pattern of ChAT loss across the five areas assayed $(\mathrm{rACg}, \mathrm{cACg}, \mathrm{LFP}$, ADL, and Hipp), as indicated by a significant group by area interaction $[F(5,30)=15.32, p<0.001]$. Post hoc analysis revealed that the anterior set of coordinates caused significant reductions compared with control only in the $\mathrm{LFP}$ and $\mathrm{CACg}$. As shown in Table 1, the pattern of reductions accompanying the lesions at the posterior site differed in that all areas assayed exhibited ChAT reductions compared with control. In order of increasing magnitude they were $\mathrm{rACg}(-21.5 \%), \mathrm{cACg}(-26.6 \%)$, Hip $(-38.4 \%)$, LFP $(-40.4 \%)$, and ADL $(-45.3 \%)$.

\section{Behavioral results}

Postoperative recovery. The lesions had no effect upon postoperative weight. Testing under the CRF schedule revealed no differences in the rate (means: sham, 17.2; anterior, 18.4; posterior, $16.5 \mathrm{rpm}$ ) or bias of responding ( $53 \%, 56 \%, 46 \%$, to the left) between the three experimental groups.

Acquisition of conditional visual discrimination. Animals with the more anterior of the two placements were significantly impaired in acquisition in terms of errors of commission. The main effect of group was significant $[F(2,16)=3.64, p<0.05]$ and resulted from the increased error score of the anterior group (mean, 1166 at $85 \%$ ) compared with the sham group [mean, $546 ; t(38)=2.65, p<0.05]$. Errors in the posterior group were quite high (mean $=890$ ) but also variable and not significantly greater than the sham group $[t(36)=1.78, p<0.1]$. Neither lesion had significant effects upon the level of omissions $[F(2,16)$ $=0.44, p>0.5]$, correct latency $[F(2,16)=0.5, p>0.5]$, or magazine latency $[F(2,16)=0.54, p>0.5]$.

\section{Correlational analysis}

To determine the relative importance of reductions in ChAT activity in the $\mathrm{CACg}$ or the hippocampus for the effects on conditional discrimination performance, the ChAT reductions were regressed separately against mean crrors madc over the last five sessions, once asymptotic performance had been attained, for all of the rats in experiments 1-3. In fact, separate regression models were generated for each of experiments $1-3$, and found not to differ significantly among themselves, allowing the data to be combined. A second model was tested to ensure the validity of collapsing the data across lesion and sham groups from experiments $1-3$. The regression coefficients of the two slopes were not significantly different $[t(52)=-1.65, p>0.1]$. An overall model could therefore be generated with the data from all three experiments, combining sham and lesion groups. The resulting linear regression was highly significant $[t(54)=-10.33$, $p<0.001]$. As shown in Figure 4, the equation of the line was $\log y=-0.21 x+1.85$, with a Pearson's $r$ of -0.82 , thus explaining approximately $67 \%$ of the variance in the data. The analysis reinforces the result suggested by the analysis of experiment 3 , that cingulate $\mathrm{Ch} \Lambda \mathrm{T}$ activity is related to accuracy of performance on the conditional discrimination task.

By comparison, when the same model was used to regress log errors against hippocampal ChAT activity there was little evi- 
dence of a strong relationship with errors (Fig. 4). Analysis of the overall regression revealed that the linear model did account for a significant proportion of the data $[t(54)=-2.93, p<$ $0.01]$. However, Pearson's $r$ was -0.45 , explaining only approximately $20 \%$ of the variance in the data, a far lower proportion compared with the cingulate model. This point is emphasized by three further considerations: (1) the slope of the line which is virtually flat $(\log y=-0.10 x+1.71)$ compared with a slope of -0.21 for errors versus ChAT activity in the $\mathrm{cACg}$ region; (2) the fact that when the correlation between ChAT activity in the cingulate cortex and hippocampus was taken into account as a contributor to the correlation between hippocampal ChAT activity and errors, the partial correlation value for the latter relationship fell further to $r=0.303$ (accounting for only $9 \%$ of the variance); and (3) the failure of the pattern of hippocampal ChAT activity to account for a significant proportion of the variance over and above the contribution of the cingulate region in a stepwise forward regression analysis. In addition, only weak relationships were found when the ChAT activity levels in the cACg were correlated with the latency measures for making a correct response or collecting earned food pellets (i.e., magazine latency), for the animals in experiments 1 and 2. This strengthens the conclusion that the lesion impairment is directly related to the ability to perform the conditional visual discrimination, rather than a breakdown in motoric or motivational processes.

\section{Experiment 4: effects of AMPA-induced lesions of the VDB}

\section{Histological analysis}

Examination of cresyl violet-stained sections of the forebrain showed that infusion of AMPA into the VDB resulted in very discrete areas of neuronal loss in the area, minor if any damage to the overlying medial septal nuclei, and without any encroachment into the lateral septal nuclei (Fig. 5). There was also very limited gliosis and minor enlargement of the lateral ventricles. Immunocytochemical visualization of $\mathrm{ChAT}$ revealed the total loss of magnocellular cholinergic neurons in the rostral VDB, but sparing of many cholinergic neurons in the most caudal aspects of the VDB and in the horizontal limb nucleus and in anterior parts of the nucleus basalis magnocellularis. This pattern of cell loss is mirrored by the regional reductions in ChAT activity measured in the experimental animals.

\section{Biochemical results}

Table 1 indicates the absolute values and percentage reductions in ChAT activity in the five brain regions analyzed. There were very substantial reductions in both the intermediate and caudal anterior cingulate samples (iACg, 70\%; $\mathrm{cACg}, 68 \%$ ), as well as reductions in the hippocampus (38\%) and LPF (40\%), with relative sparing of the $\mathrm{rACg}(19 \%)$. This selective pattern of effect was confirmed by finding a significant group $\times$ region interaction in the ANOVA. Post hoc analyses of the simple main effects of group showed that only the reduction in the $\mathrm{rACg}$ was not significant, and that the reductions in the $\mathrm{iACg}$ and $\mathrm{cACg}$ samples were significantly greater than in the other areas.

Two distinct subgroups of rats were readily identifiable from an examination of the individual data. Several rats had reductions in ChAT activity largely limited to the cingulate cortex as compared to other areas, whereas other rats showed evidence of more widespread reductions. On the basis of this post hoc separation, two equal subgroups were formed, cingulate-selec-

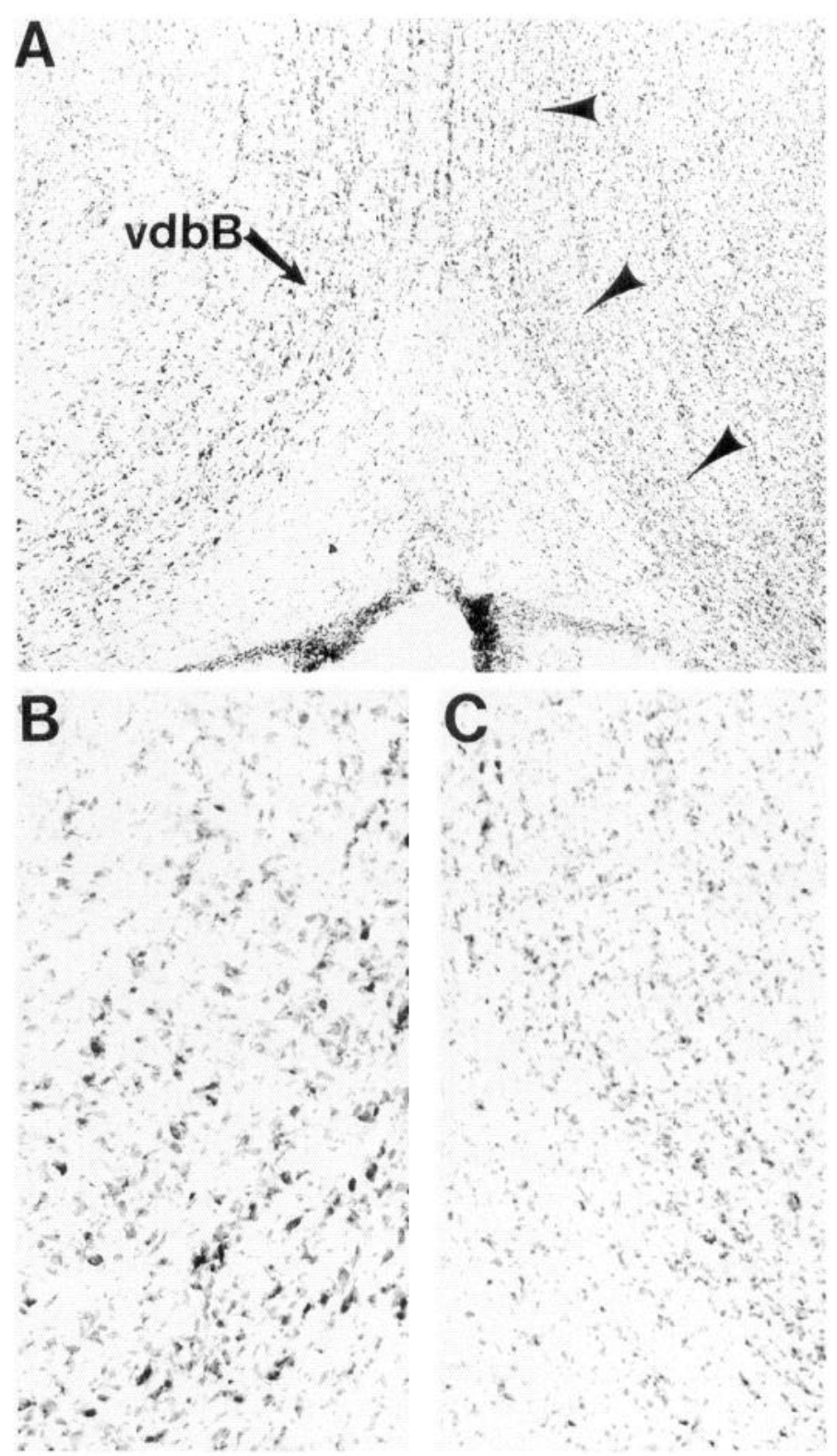

Figure 5. Photomicrograph of the ventral medial septum and the vertical limb nucleus of the diagonal band of Broca $(\mathrm{VdbB})$, showing the effects of a unilateral AMPA-induced lesion. On the left hand side of $A$ the normal appearance of the VDB can be seen, with many large neuronal profiles visible. $B$, shows an enlargement of the normal VDB seen in $A$. On the right in $A$, the consequences of infusing AMPA are clearly seen. There is total loss of large neuronal profiles (indicated along the line of arrowheads), and this is very clearly seen in the enlargement in $C$. Magnification: $A, 50 \times ; B$ and $C, 130 \times$.

tive and nonselective, both $n=7$. Following this reassignment, the mean reductions in ChAT activity were as follows (cingulateselective group first): $\mathrm{rACg}, 7 \%, 30 \%$; $\mathrm{iACg}, 63 \%, 74 \%$; $\mathrm{ACg}$, $60 \%, 72 \%$; hippocampus, $23 \%$, $54 \%$; LPF, $24 \%, 56 \%$. ANOVA of the raw data confirmed that the cingulate subgroup exhibited significant reductions in ChAT activity only in the iAC and $\mathrm{oAC}$. Further analysis showed that the reductions in iACg and $\mathrm{cACg}$ did not differ between the two subgroups, but that there were significantly greater reductions in ChAT activity in the nonselective group for all of the remaining regions. 


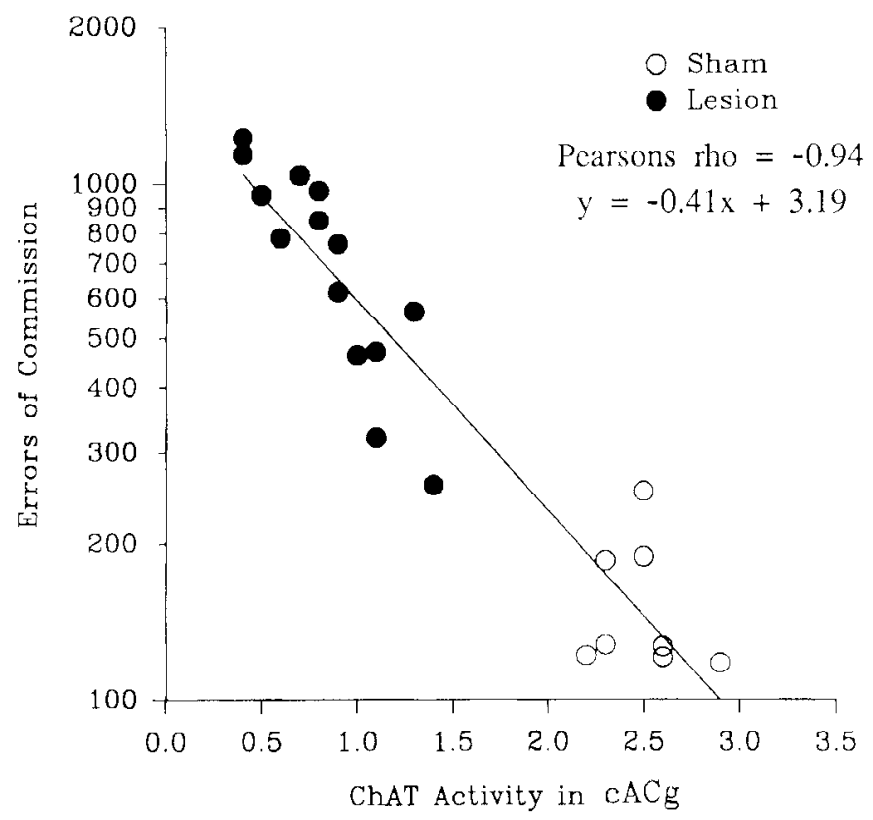

Figure 6. Log linear regression of errors of commission reattaining the $75 \%$ correct criterion and ChAT activity in the $\mathrm{ACg}$ for experiment 4. Each point represents an individual rat. Also shown are the equations of the best-fitting line and values for Pearson's $r$.

\section{Behavioral results}

Postoperative recovery. At the end of the recovery period, there were no significant differences among the groups in body weight (sham, $289 \mathrm{gm}$; ACg-selective, $279 \mathrm{gm}$; nonselective, $274 \mathrm{gm}$ ). Upon return to the CRF schedule, there were again no differences among the three groups in rate of responding (sham, 10.5; ACg-selective, 8.9 ; nonselective, $9.2 \mathrm{rpm}$ ) or in spatial bias $(52.8 \%, 49.5 \%, 54.8 \%$, to the left $)$.

Reacquisition of the conditional visual discrimination. In the initial $10 \mathrm{~d}$ testing period, the two lesion subgroups were significantly less accurate than the sham control group, but did not differ significantly from one another [group effect, $F(2,18)=$ $18.68, p<0.001]$. There was also a significant group $\times$ sessions interaction $[F(2,162)=1.89, p<0.025]$, resulting from a slower rate of recovery in the two lesion subgroups after session 1 , as compared to the sham group. However, there were no differences in errors of omission, or in latencies to respond correctly or to visit the magazine following reinforcement among the three groups ( $F<1.2$ in all cases). Analysis of spatial lever bias did reveal significant differences among the three groups $[F(2,162)$ $-7.62, p<0.05]$. The nonselective group showed a significant bias to responding to the slow stimulus that averaged $67 \%$ over the 10 days and did not significantly change over this period. The sham and $\mathrm{ACg}$-selective groups showed no significant bias (57\% and $51 \%$ to slow, respectively).

The two lesion subgroups were significantly impaired in the reacquisition of the discrimination to a $75 \%$ correct criterion of learning, with both groups requiring more errors of commission to reach this value than the sham group $[F(2,18)=11.93, p<$ $0.01]$, but not differing from one another. Mean values were sham, 168.3; ACg-selective, 627.7; nonselective, 866.6. Asymptotic levels of performance were equivalent between the groups.

\section{Correlational analysis}

Regression analysis was applied to the relationship between ChAT activity and log errors of commission to reach the criterion of relearning to $75 \%$ correct. Separate analyses were run for ChAT activity in the $\mathrm{CACg}$ region, $\mathrm{rACg}$, the $\mathrm{LFP}$, and hippocampus.

Regression coefficients were almost identical between the sham and lesion groups for each region and so data were combined in each case for separate regression analyses. In all arcas except for $\mathrm{rACg}$, ChAT activity was significantly $(p<0.001)$ inversely related to errors of commission (Pearson's $r \mathrm{cACg},-0.94$; LFP,

-0.85 ; hippocampus, $-0.71 ; \mathrm{rACg},-0.30$; all $\mathrm{df}=20$ ). The regression for the $\mathrm{CACg}$ shown in Figure 6 thus accounted for approximately $89 \%$ of the variance $[t(19)=5.34, p<0.001]$. Reductions in ChAT activity were highly intercorrelated among the various regions, and so partial correlations were computed to reduce possible resulting artefactual associations. The only area to retain a significant correlation with log errors, following this allowance for the variance attributable to the remaining areas, was the $\mathrm{cACg}$. Thus, for example, the partial correlation coefficients relating errors to hippocampal and LFP ChAT activity were as low as -0.32 and -0.29 , respectively, when the relationship to ChAT activity in $\mathrm{cACg}$ was taken into account. A stepwise forward regression analysis also confirmed that the ChAT activity reductions in the $\mathrm{ACg}$, LFP, or the hippocampus failed to account for a significant proportion of the variance over and above that contributed by the $\mathrm{cACg}$ region. These results reinforce the results of similar analyses made in experiments 1-3 (Fig. 4), with a different group of rats, a slightly different task, and measure of performance (errors to reattain a learning criterion) as well as a different method for inducing excitotoxic lesions of the VDB.

\section{Discussion}

Each of the four experiments showed that lesions of the septum and VDB produced a significant deficit in either the acquisition or the reacquisition (experiment 4 ) of a conditional visual discrimination. Although the methods used to induce the lesions were presumably not specific for cholinergic neurons, there was nevertheless a very high correlation between errors of commission and ChAT activity in certain regions of the cingulate cortex. These expcriments therefore suggest that these cholinergic projections have an important role in the mediation of conditional discrimination performance.

A conditional discrimination task requires that an animal learns specific decision rules to guide response sclection. The conditional nature of the discrimination (i.e., if stimulus a, then perform response $\mathrm{x}$; if stimulus $\mathrm{b}$, then perform response $\mathrm{y}$ ) means that the discrimination cannot be solved by physically approaching the rewarded stimulus, as is the case with simple simultaneous discriminations. Lesions similar to those studied here failed to impair the learning or performance of simple brightness discriminations (Marston et al., 1993). Therefore, it is likely that these deficits reflect a considerable degree of behavioral specificity, being related to mechanisms governing response selection or choice. This conclusion is substantiated by other behavioral measures taken in this task. Thus, the latencies to respond correctly or to collect earned food pellets were not affected by the lesions, nor did errors of omission increase. Furthermore, preliminary testing under a schedule of continuous reinforcement was never significantly affected in any of the 
four experiments. Consequently, it is most unlikely that the present deficits can be accounted for by simple sensory, motivational, or motor impairments.

\section{Specificity of the effects of the lesions}

The lesions were made by infusing the excitotoxins quisqualic acid or AMPA. Previous work on the effects of excitotoxic lesions of the septum has shown that it is possible to produce effective lesions using ibotenic acid, which result in significant reductions in hippocampal ChAT activity (e.g., Hagan et al., 1988). However, in preliminary experiments the use of ibotenic acid led to a high rate of mortality and considerable debilitation of the animals, as well as difficulty in controlling the tendency for the neurotoxin to damage the lateral, as well as medial septum. Moreover, in our previous investigations of the functions of the cholinergic projection from the nbm, we found that quisqualate and AMPA were both more effective in reducing cortical ChAT activity and less likely to damage noncholinergic neurons within the basal forebrain, especially in the pallidum (Page et al., 1991; see also Sarter and Dudchenko, 1991). This general conclusion has been confirmed in the present experiments, where the histological analysis provided for experiment 4 confirms that AMPA can be used to produce profound reduction in cortical cholinergic markers without producing gross nonspecific damage in the area of infusion.

The effects of the various excitotoxic lesions on ChAT activity in experiments $1-4$ are compatible with a number of retrograde tracing studies (Bigl et al., 1982; Saper, 1984; Gaykema et al., $1990,1991)$ that support the concept that cell bodies from the nbm, VDB, and medial septum form a continuum of topographically organized but overlapping projections to the cerebral cortex. Those found most rostrally, in the septal nucleus, project to the ventral hippocampus and cingulate cortex. More caudally and latcrally, the VDB projects to that portion of the cingulate cortex caudal to the genu of the corpus callosum (Gaykema et al., 1990, 1991) and to the dorsal hippocampus, while the nbm and HDB project to neocortical regions and the olfactory bulb, respectively.

Our experimental approach evolved over the course of the four experiments. In experiments $1-3$ we employed quisqualic acid, and in experiment 4 , in the light of our subsequent discoveries concerning its efficacy when lesioning the nbm (Page et al., 1991), AMPA itself. Initially, we were concerned with obtaining substantial effects on the hippocampal and cingulate cortex cholinergic projections and hence large lesions of the septum were made, using quisqualate. This lesion was nevertheless less debilitating for the rats than that produced by ibotenic acid in pilot experiments. It also resulted in substantial reductions in ChAT activity in both the hippocampus and the cingulate cortex without producing any significant effects on a major projection region of the $\mathrm{nbm}$ (anterior dorsolateral cortex) or on levels of forebrain monoamines or their metabolites. The pattern of effect on cortical ChAT is thus consistent with damage to the VDB, which sends a rich cholinergic projection to the cingulate cortex. Consequently, in experiment 2 , smaller quisqualate-induced lesions of the medial septum were studied, resulting, as expected, in a more variable pattern of ChAT reductions in the hippocampus and cingulate cortex which necessitated a post hoc reassignment of animals to two subgroups. The results of both experiments 1 and 2 implicated cholinergic reductions in the cingulate cortex rather than the hippocampus (or terminal regions receiving cholinergic projec- tions from the nbm) in the behavioral deficits. Thus, in experiment 3, we compared explicitly the effects of anterior and posterior placements in the region of the diagonal band of Broca. The behavioral deficits were considerably greater in the former group, where the ChAT activity reduction was largely limited to the cingulate, and certain regions of the frontal cortex. By contrast, the more posterior placement produced reductions in ChAT activity in the anterior dorsolateral neocortex, presumably due to damage to neurons of the anterior nbm, but had relatively little effect on the conditional discrimination. Conditional discrimination deficits have also been found in marmosets following excitotoxic lesions aimed at the septum/VDB (Ridley et al., 1989), although cingulate ChAT activity was not measured in that study. The correlation between hippocampal ChAT and errors (partial correlation, $r=-0.30$ ) found in the present experiments 1-3 was almost the same as for experiment 4 , but the partial correlation of $r=-0.32$ in the latter experiment was not significant. I Ience, these experiments support the conclusion that the cholinergic innervation of the hippocampus is less important than the cholinergic innervation of the cingulate cortex in the mediation of this conditional discrimination. This conclusion is consistent with the relative lack of effect of excitotoxic lesions of the hippocampus on acquisition of the same task as used here (Marston et al., 1993).

The consistency of the results over experiments $1-3$ enabled a regression model to be developed of the results relating level of ChAT activity exponentially to the errors of commission made on the discrimination once asymptotic performance had been reached for lesioned and sham animals combined. This exponential relationship was by far the strongest for the perigenual and postgenual regions of cingulate cortex, accounting for almost $70 \%$ of the variance over the three experiments. This represents an extraordinarily high correlation for experiments of this nature, based on data obtained from 57 animals in all; the presence of the significant relationship even in sham-operated rats also was noteworthy. However, further work will be required to determine the precise regions of the cingulate cortex that are implicated in the effect. For example, the rostral portions of the anterior cingulate showed no particular relationship with task performance, and it is likely that the strong correlation found for its caudal aspects would have extended to the posterior cingulate cortex. Indeed, some of the tissue actually assayed as postgenual may well contribute to the anterior region of the posterior cingulate cortex (Brodmann area 29) because it receives projections of the anteroventral and anterodorsal thalamic nuclei (Vogt, 1985). The possible role of the posterior cingulate is strengthened by our recent unpublished findings $(\mathrm{J}$. Muir, T. Bussey, T. W. Robbins, and B. J. Everitt) that excitotoxic lesions of the posterior, but not the anterior, cingulate impair the later learning stages of the same conditional discrimination as used here.

The very high correlation of the accuracy of task performance with cingulate ChAT activity strongly suggests that the lesions produced their effects by impairing transmission in the cholinergic system, particularly as measures of monoamine levels showed no changes in experiment 1 . The conclusion was supported by the finding of an even stronger relationship $(r=-0.94)$ between ChAT activity and the errors committed in reattaining criterion following AMPA-induced lesions aimed at the caudal VDB in experiment 4 . Of course, it would be bolstered still further by demonstrations of pharmacologically specific reversals of the behavioral deficits, and this is the subject of current 
work. However, it is still logically possible for the relationship to result from damage to some other system, for example, to GABAergic projections arising from the septum and VDB that also innervate the hippocampus and cingulate cortex (Köhler et al., 1984).

\section{Nature and implications of the behavioral deficit}

The results from experiments 1 and 2 taken together suggest that the deficit exhibited by the lesion group was not primarily one of learning, but rather an inability to perform the task optimally. If the deficit had been in learning, then the slope of the acquisition curve of the lesioned group would have been flatter, though eventually reaching the same asymptote. The converse was the case, the curves being initially superimposed before the lesion group stabilized at a lower asymptote. Experiment 4 confirmed that rats exhibited a performance deficit when given an AMPA-induced lesion after training on a very similar discrimination. They reattained the discrimination to previous levels of performance on average in considerably fewer sessions than it took initially to learn the discrimination and, as pointed out above, there was little evidence that the impairment was a result of disrupted motivational and motoric processes. Experiment 4 also controlled for the possibility that the rats were utilizing flux cues in making the visual discrimination; thus, the deficit appears to be one of failure to relate cues of temporal frequency conditionally to specific responses.

The results add to the rather sparse set of published data on the functions of the cingulate cortex in the rat. Anatomical, behavioral, and electrophysiological evidence compels the conclusion that the functions of the anterior and posterior cingulate cortex are interactive, but quite distinct, with the latter being linked to the functions of the hippocampal formation and posterior association cortex (Gabriel et al., 1980; Buchanan and Powell, 1982; Kolb, 1984; Vogt, 1985). Thus, lesions of the hippocampus or the fimbria-fornix produce comparable deficits on spatial reference and memory tasks to those limited to the posterior cingulate cortex (Sutherland et al., 1988). On the other hand, aspirative lesions of the posterior cingulate or retrosplenial cortex have been reported to spare several forms of conditional discrimination, where objects must be related to spatial, directional, or other contextual cues (Markowska et al., 1988). However, lesions made in this way will damage afferent fibers to the anterior cingulate cortex, and aspirative lesions of the latter will similarly disrupt afferents to the posterior cingulate.

In a notable series of studies employing an electrophysiological analysis of discriminated avoidance conditioning in rabbits, discriminative neuronal activity has been shown to develop earlier in training in the anterior than the posterior cingulate, leading to the suggestion that the latter area is more concerned with the maintenance of the avoidance response than its acquisition (Gabriel, 1991; Gabriel et al., 1991). This hypothesis might now be extended by the results of the present study where highly significant and selective correlations between accuracy of appetitively motivated discrimination performance and indices of cholinergic activity have been demonstrated in the cingulate cortex. The apparent anatomical specificity of this relationship, taken in conjunction with other results (e.g., Robbins et al., 1989a,b; Muir et al., 1992), suggests that the cortical cholinergic projections contribute to different behavioral functions, mediated by different cortical regions.

\section{References}

Bigl V, Woolf NJ, Butcher LL (1982) Cholinergic projections from the basal forebrain to frontal, parietal, temporal, occipital and cingulate cortices: a combined fluorescent tracer and acetylcholinesterase analysis. Brain Res Bull 8:727-749.

Bowen DM, Smith CB, White P, Davison AN (1976) Neurotransmitter related enzymes and indices of hypoxia in senile dementia and other bioatrophies. Brain 99:459-496.

Buchanan SL, Powell DA (1982) Cingulate cortex: its role in Pavlovian conditioning. J Comp Physiol Psychol 96:755-774.

Dunnett SB (1985) Comparative effects of cholinergic drugs and lesions of nucleus basalis of fimbria-formix on delayed matching in rats. Psychopharmacology 87:357-363.

Dunnett SB, Everitt BJ, Robbins TW (1991) The basal forebraincortical cholinergic system: interpreting the functional consequences of excitotoxic lesions. Trends Neurosci 14:494-501.

Etherington R, Mittleman G, Robbins TW (1987) Comparative effects of nuclcus basalis and fimbra-fornix lesions on delayed matching and alternation tests of memory. Neurosci Res Commun 1:135-143.

Everitt BJ, Robbins TW, Gaskin M, Fray PJ (1983) The effects of lesions to ascending noradrenergic neurons on discrimination learning and performance in the rat. Neuroscience 10:397-419.

Everitt BJ. Robbins TW, Evenden JL. Marston HM, Jones GJ, Sirkia TE (1987) The effects of excitotoxic lesions on the substantia innominata, ventral and dorsal globus pallidus on the acquisition and retention of a conditional visual discrimination: implications for cholinergic hypotheses of learning and memory. Neuroscience 22:441469.

Fonnum F (1975) A rapid radinchemical method for the determination of choline acetyltransferase. J Neurochem 24:407-409.

Gabriel M (1991) Functions of the anterior and posterior cingulate cortex during avoidance conditioning in rabbits. In: Progress in brain research, Vol 85, The prefrontal cortex, its structure, function and pathology (Uylings HBM, Van Eden CG, de Bruin JPC, Feenstra MGP, Corner MA, Eikelboun T, Janssen A, eds), pp 457-473. Ansterdam: Elsevier/North-Holland.

Gabricl M, Foster K, Orona E, Saltwick SE, Stanton M (1980) Neuronal activity of cingulate cortex, anteroventral thalamus and hippocampal formation in discriminative conditioning: encoding and extraction of the significancc of conditional stimuli. In: Progress in psychobiology and physiological psychology, Vol 9 (Sprague J, Epstein AN, eds), pp 126-233. New York: Academic.

Crabriel M, Kuhota Y, Sparenborg S, Straube K, Vogt BA (1991) Effects of cingulate cortical lesions on avoidance learning and training-induced unit activity in rabbits. Exp Brain Res 86:585-600.

Gaykema RPA, Luiten PGM, Nyakas C, Traber J (1990) Cortical projection patterns of the medial septum-diagonal band complex. J Comp Neurol 293:103-124.

Gaykema RPA, Van Weghel R, Hersh LB, Luiten PGM (1991) Prefrontal cortical projections to the cholinergic neurons in the basal forebrain. J Comp Neurol 303:563-583.

Hagan JJ, Salamone JD, Simpson J, Iversen SD, Morris RGM (1988) Place navigation in rats is impaired by lesions of medial septum and diagonal band but not nucleus basalis magnocellularis. Behay Brain Res 27:9-20.

Hepler DJ, Olton DS, Wenk GL, Coyle JT (1985) Lesions in nucleus basalis magnocellularis and medial scptal area of rats produce qualitatively similar memory impairments. J Neurosci 5:866-873.

Hodges H, Allen Y, Sinden J, Lantos PL, Gray JA (1991) The effects of cholinergic-rich neural grafts on radial maze performance of rats after excitotoxic lesions of the forebrain cholinergic projection system. II. Cholinergic drugs as probes to investigate lesion-induced deficits and transplant-induced functional recovery. Ncuroscience 45:609623.

Jones GH, Hernanadez TD, Kendall DA, Marsden CA, Robbins TW (1992) Dopaminergic and serotonergic function following isolation rearing in rats: study of behavioural responses and postmortem and in vivo neurochemistry. Pharmacol Biochem Behav 43:17-35.

Köhler C, Chan-Palay V, Wu J (1984) Septal neurons containing glutamic acid decarboxylase immunoreactivity project to the hippocampal region in the rat brain. Anat Embryol (Berl) 169:41-44.

Kolb B (1984) Functions of the frontal cortex of the rat. Brain Res Rev 8:65-98.

Markowska AL, Olton DS, Murray EA, Gaffan D (1988) A compar- 
ative analysis of the role of the fornix and cingulate cortex in memory: rats. Exp Brain Res 74:187-201.

Marston HM, Everitt BJ, Robbins TW (1993) Comparative effects of excitotoxic lesions of the hippocampus and septum/diagonal band on conditional visual discrimination and spatial learning. Neuropsychologia 31:1099-1118

Mefford IN (1981) Application of high performance liquid chromatography with electrochemical detection to neurochemical analysis: measurements of catecholamines, serotonin and metabolites in the rat brain. J Neurosci Methods 3:207-224.

Morris RGM, Garrud P, Rawlins JNP, O'Keefe J (1982) Place navigation impaired in rats with hippocampal lesions. Nature 297:681583.

Muir JL, Dunnett SB, Robbins TW, Everitt BJ (1992) Attentional function of the forebrain cholinergic system: effects of intraventricular hemicholinium, basal forebrain lesions and intracortical grafts on a multiple choice serial reaction time task. Exp Brain Res 89:611-622.

Murray CL, Fibiger HC (1985) Learning and memory deficits after lesions of the nucleus basalis magnocellularis: reversal by physostigmine. Neuroscience 14:1025-1032.

Olton DS, Givens BS, Markowska AL, Shapiro M, Golski S (1991) Mnemonic functions of the septohippocampal cholinergic system. In: Memory: organization and locus of change (Squire LR, Weinberger NM, Lynch G, McGaugh JL, eds), pp 250-269. New York: Oxford UP.

Page KJ, Everitt BJ, Robbins TW, Marston HM, Wilkinson LJ (1991) Dissociable effects on spatial maze and passive avoidance acquisition and retention following AMPA and ibotenic acid excitotoxic lesions of the basal forebrain in rats: differential dependence on cholinergic neuronal loss. Neuroscience 43:457-472.

Paxinos G, Watson C (1986) The rat brain in stereotaxic coordinates, $2 d$ ed. Sydney: Academic.

Perry EK, Curtis M, Dick DJ, Candy JM, Atack JR, Bloxham CA, Blessed G, Fairbairn A, Tomlinson BE, Perry RH (1985) Cholinergic correlates of cognitive impairments in Parkinson's disease: comparisons with Alzheimer's disease. J Neurol 43:413-421.

Ridley RM, Murray TK, Johnson JA, Baker HF (1986) Learning impairment following lesion of the basal nuclcus of Meynert in the marmoset: modification by cholinergic Grugs. Brain Res 376:108116.

Ridley RM, Aitken DM, Baker HF (1989) Learning about rules but not about reward is impaired following lesions of the cholinergic projection to the hippocampus. Brain Res 502:306-318.

Robbins TW, Everitt BJ, Ryan CN, Marston HM, Jones GJ, Page KJ (1989a) Comparative effects of quisqualic and ibotenic acid-induced lesions of the substantia innominata and globus pallidus on the acquisition of a conditional visual discrimination: differential effects on cholinergic mechanisms. Neuroscience 28:337-352.

Robbins TW, Everitt BJ, Marston HM, Wilkinson J, Jones GH, Page KJ (1989b) Comparative effects of ibotenic acid-and quisqualic acid-induced lesions of the substantia innominata on attentional function in the rat: further implications for the role of the cholinergic neurons of the nucleus basalis in cognitive processes. Behav Brain Res 35:221-240.

Saper CB (1984) Organization of cerebral cortical afferent systems in the rat. I. Nucleus basalis magnocellularis. J Comp Neurol 222:311342.

Sarter M, Dudchenko P (1991) Dissociative effects of ibotenic and quisqualic acid induced basal forebrain lesions on cortical acetylcho linesterase-positive fiber density and cytochrome oxidase activity. Neuroscience 41:729-738

Sutherland R J, Whishraw IQ, Kolb B (1988) Contributions of cingulate cortex to two forms of spatial learning and memory. J Neurosci 8:18631872 .

Vogt BA (1985) The cingulate cortex. In: Cerebral cortex, Vol 4, Association and auditory cortices (Jones EG, Peters A, eds), pp 89-149. New York: Plenum.

Wenk GL, Markowska AL, Olton DS (1989) Basal forebrain lesions and memory. Alterations in neurotensin, not acetylcholine, may cause amnesia. Behav Neurosci 103:765-769.

Whishaw IQ, O'Connor WT, Dunnett SB (1985) Disruption of central cholinergic systems in the rat by basal forebrain lesions or atropine: effects on feeding, sensorimotor behaviour, locomotor activity and spatial navigation. Behav Brain Res 17:103-115.

Zilles K (1985) The cortex of the rat. Berlin: Springer. 